\title{
Dificultades y controversias en el manejo hospitalario de la hemorragia digestiva baja
}

\author{
L. Martín Martín, M. Trapero-Marugán, J. Cantero Perona y R. Moreno Otero \\ Servicio de Hepatogastroenterología y Centro de Investigación Biomédica en Red de Enfermedades Hepáticas y \\ Digestivas (CIBERehd). Hospital Universitario de La Princesa. Universidad Autónoma de Madrid
}

\section{RESUMEN}

Objetivos: la hemorragia digestiva baja (HDB) es una causa frecuente de ingreso hospitalario; pese a ello, no se conocen con exactitud los factores que influyen en su evolución. Los objetivos de este trabajo fueron comparar los cambios en el manejo de la HDB en nuestro Servicio entre los años 2005 y 2007, así como analizar diferentes parámetros que pudieran influir en su pronóstico.

Pacientes y métodos: se incluyeron retrospectivamente todos los ingresos por HDB durante el periodo 2005-2006 y prospectivamente los del 2006-2007. En todos se realizó historia clínica, exploración -incluyendo tacto rectal- y análisis sanguíneo. Se realizó colonoscopia en la mayoría de los pacientes.

Resultados: se incluyeron 137 pacientes en el 2005-2006: requirieron transfusión de hemoderivados el 36\%. El 31\% había presentado algún episodio de HDB previamente. El 62\% presentó una evolución favorable. El tiempo desde el ingreso hasta la colonoscopia y la estancia media fueron de 4,1 y 10,2 días respectivamente. En el 2006-2007 se incluyeron 96 pacientes: requirieron transfusión el 42\%. El 33\% había presentado HDB previamente. La evolución fue favorable en el $67 \%$. El tiempo hasta la colonoscopia y la estancia media fueron de 2,6 y 7,7 días respectivamente. Los divertículos fueron el hallazgo más frecuente en ambos periodos.

Conclusiones: durante el 2006-2007 la estancia media de los pacientes con HDB ingresados en el Servicio de Aparato Digestivo se redujo respecto al 2005-2006 en un 25\% y el tiempo de realización de la colonoscopia en un $37 \%$; esto no logró más localizaciones del punto sangrante ni una disminución en la recurrencia de la hemorragia.

Palabras clave: Hemorragia digestiva baja. Colonoscopia. Comorbilidad. Estancia media hospitalaria.

Agradecimientos: CIBERehd está patrocinado por el Instituto de Salud Carlos III.

Recibido: $07-05-08$

Aceptado: 08-07-08.

Correspondencia: Leticia Martín Martín. Servicio de Aparato Digestivo. Hospital Universitario de La Princesa. Diego de León, 62. 28006 Madrid. e-mail:1mleticia@yahoo.es; mtraperomarugan@hotmail.com

\begin{abstract}
Objectives: lower intestinal bleeding (LGIB) is a frequent reason for hospitalization; however, the prognostic factors have not been clearly defined. The aim of this paper was to analyze several clinical parameters and the management of this entity in our department from 2005 to 2007.

Material and methods: all hospitalized patients with LGIB were retrospectively (2005-2006) and prospectively (2006-2007) included. Medical records, physical examination (anal digital examination included), blood testing, and colonoscopic examination (in most of patients) were performed.

Results: 137 patients were included during 2005-2006: 36\% of them required blood transfusion; thirty-one percent of patients showed previous episodes of LGIB, and $62 \%$ had a favorable outcome. Time from admission to colonoscopy was 4.1 days, and length of stay was 10.2 days. In the 2006-2007 study 96 patients were included: $42 \%$ of them required blood transfusion, thirtythree percent of patients showed previous episodes of LGIB, and $68 \%$ had a favorable outcome. Time from admission to colonoscopy was 2.6 days, and length of stay was 7.7 days. The most frequent etiology was diverticulosis in both studies.

Conclusions: hospital length of stay and time from admission to colonoscopy in patients with LGIB was reduced by $25 \%$ and $37 \%$, respectively, in the 2005-2006 period with regard to the 2006-2007 one; however, there were no more bleeding points or a decrease in bleeding recurrence.
\end{abstract}

Key words: Lower intestinal bleeding. Colonoscopy. Comorbidity. Length stay.

Martín Martín L, Trapero-Marugán M, Cantero Perona J, Mo reno Otero $R$. Dificultades y controversias en el manejo hospitalario de la hemorragia digestiva baja. Rev Esp Enferm Dig 2008; 100: 560-564. 


\section{INTRODUCCIÓN}

Las hemorragias digestivas son la causa más frecuente de ingreso en las unidades de gastroenterología y aproximadamente una cuarta parte de ellas son de origen bajo (1). La incidencia anual de la hemorragia digestiva baja (HDB) se sitúa entre 20,5 y 27 casos hospitalizados por 100.000 habitantes frente a los 100-200 casos por 100.000 habitantes de hemorragia digestiva alta (2).

Nuestro hospital tiene una población asignada de 468.000 habitantes. En el año 2005 el 10,7\% de los ingresos en el Servicio de Aparato Digestivo fueron por HDB y en el 2006 esta fue causa del $8 \%$ de los ingresos.

Los factores que influyen en la evolución de la hemorragia digestiva alta (HDA) son bien conocidos, pero disponemos de menos información sobre las causas que influyen en la presentación y evolución de la HDB; de ahí la tendencia más o menos generalizada a ingresar de forma rutinaria a todos aquellos pacientes que acuden al Servicio de Urgencias con una HDB, excepto en los casos en los que la sospecha es el origen anorrectal. Con esta actitud probablemente originemos ingresos no estrictamente necesarios, y por tanto, un aumento en el gasto sanitario. Este estudio se inició de forma retrospectiva (2005-2006) con el objetivo de analizar los factores que pudieran influir en la evolución y el pronóstico de la HDB, la carga asistencial que suponía para el Servicio de Aparato Digestivo, así como analizar la forma en que se maneja esta patología. Simultáneamente se diseñó un estudio prospectivo (2006-2007) durante el año consecutivo, previa modificación de la pauta de actuación hasta entonces llevada a cabo en los casos de HDB. Para ello, debíamos contar con una Unidad de Endoscopia que permitiese la realización de la colonoscopia de una forma precoz.

La HDB se define como aquella que se origina distalmente al ángulo de Treitz; siendo el 90\% de origen cólico (3). Su presentación clínica es muy variable, desde la hematoquecia con mínima cantidad de sangre hasta la hemorragia masiva con shock. Son menos graves que las HDA y tienden a ser autolimitadas en el $80 \%$ de los casos (4). La mortalidad es menor del 5\% y generalmente se asocia a comorbilidad. Esta, los antiinflamatorios no esteroideos (AINE), las necesidades transfusionales y la inestabilidad hemodinámica al ingreso son factores de riesgo para morbimortalidad (5-7).

La incidencia de la HDB es mayor en hombres y aumenta con la edad debido a la mayor prevalencia de diverticulosis y patología vascular que son las principales causas de HDB en ancianos (2). Menos frecuentes son las neoplasias, el sangrado postpolipectomía (entre el 0,2 y el $0,6 \%$ de las polipectomías) (8-10), las colitis, la proctitis actínica, las lesiones del intestino delgado, el Dieulafoy, las varices rectales, la úlcera rectal solitaria (puede causar hasta el 16,4\% de las HDB) (12) y la patología hemorroidal.
Hasta hace poco la colonoscopia se ha utilizado como procedimiento diagnóstico en la HDB de forma programada, pero en los últimos años varios estudios han evaluado la rentabilidad de la colonoscopia urgente frente a otras técnicas, fundamentalmente radiológicas: estudios retrospectivos concluyen que la realización de la colonoscopia urgente es factor predictivo independiente de disminución de la estancia media hospitalaria $(7,13)$ y otros aleatorizados no encuentran diferencias entre la realización de la colonoscopia urgente y la angiografía intervencionista con colonoscopia posterior programada (14). La colonoscopia urgente permite obtener el diagnóstico específico en un elevado porcentaje de pacientes y además es potencialmente terapéutica por lo que es el tratamiento de elección en la mayoría de los casos de HDB $(13,15,16)$; cuando esta aporta resultados indeterminados o no se puede realizar, las técnicas radiológicas son fundamentales (17).

Uno de los aspectos que despierta mayor interés es la influencia de la anticoagulación y antiagregación en la HDB. Se estima que en Estados Unidos 60 millones de personas consumen antiinflamatorios (AINE). En el caso de la HDA su uso ha sido intensamente estudiado; no sucede lo mismo con la HDB. Se podría esperar el mismo riesgo de sangrado por un mecanismo similar de daño en la mucosa cólica que en las hemorragias altas; sin embargo estudios observacionales sugieren un menor número de complicaciones asociadas a AINE en la HDB respecto a la HDA (18). No existen estudios que analicen y relacionen directamente la terapia con anticoagulantes y HDB.

\section{PACIENTES Y MÉTODOS}

Se incluyeron de forma prospectiva todos los pacientes ingresados con diagnóstico de HDB en el Servicio de Aparato Digestivo de nuestro hospital en el periodo de junio de 2006 a junio de 2007; y de forma retrospectiva mediante la revisión de las historias clínicas, todos los pacientes que habían ingresado con el mismo diagnóstico entre junio de 2005 y junio de 2006.

En todos los pacientes se realizó la historia clínica y exploración física al ingreso incluyendo tacto rectal y un análisis sanguíneo (hemograma, bioquímica y coagulación). Se recogieron edad, sexo, consumo de tabaco y alcohol (consideramos consumo significativo a partir de 20 gramos diarios), comorbilidad asociada - presencia de al menos dos factores de riesgo cardiovascular (uno si se trataba de diabetes mellitus), historia previa o actual de eventos cardiovasculares, enfermedad pulmonar obstructiva crónica, patología renal, hepatopatía etc.-, consumo de AINE (se incluyeron tanto los pacientes que los consumían diariamente, como su uso puntual en los 15 días previos al ingreso), tratamiento anticoagulante (e INR en urgencias) y las cifras de hemoglobina y hematocrito en el momento del ingreso y en el alta. Se registraron episo- 
dios previos de HDB y de HDA y se interrogó acerca de las características y ritmo de las deposiciones, presencia de dolor abdominal, síncope o síndrome constitucional. Se documentó también la necesidad de transfusión de hemoderivados.

En la inspección anal se observó la existencia de fisuras, fístulas o hemorroides y en el tacto rectal la presencia o ausencia de masas y el contenido en la ampolla (normal o con sangrado; y si este fue en forma de melena, hematoquecia o sangre roja).

Durante el ingreso se realizó colonoscopia, previo consentimiento informado, a la mayoría de los pacientes, con excepción de aquellos que habían sido sometidos a una colonoscopia previa reciente o en los que se descartó la realización de una prueba invasiva. Se recogió el día del ingreso en el que se realizó la colonoscopia, considerándola urgente si esta se hizo durante el primer día. Los resultados de la colonoscopia se describieron según la localización y la patología. Se registraron también los casos en los que se realizó terapéutica endoscópica y el método empleado (electrocoagulación, esclerosis, argón plasma o hemoclips).

La evolución del sangrado fue clasificada como "favorable", cuando la hemorragia cesó de forma espontánea en menos de 72 horas; "persistente", en los casos en los que el sangrado no cesó totalmente en 72 horas; "recurrente", en los casos en los que el paciente había presentado un episodio previo reciente de HDB, o lo presentó posteriormente.

\section{Análisis estadístico}

Para realizar el estudio estadístico se utilizó el paquete estadístico SPSS versión 12 en español. Se utilizaron los test de t-Student, ANOVA y regresión logística para las variables cuantitativas y para las cualitativas Chi cuadrado con las correcciones pertinentes (Fisher, Yates). Valores de $\mathrm{p}<0,05$ y $\mathrm{F}<0,05$ se consideraron estadísticamente significativos. El protocolo fue aprobado por el comité ético del hospital.

\section{RESULTADOS}

Se incluyeron 233 pacientes, que representan el número total de ingresos por HDB en el Servicio de Aparato Digestivo de nuestro hospital durante 2 años; 137 de forma retrospectiva (junio 2005-junio 2006) y 96 de forma prospectiva (junio 2006-junio 2007). Se llevó a cabo el análisis estadístico en cada uno de los grupos, así como una comparación entre ambos.

\section{Estudio retrospectivo: 2005-2006}

De los 137 pacientes incluidos, 66 fueron hombres y 71 mujeres. La edad media fue de 73,5 (24-95) años. Pre- sentaron datos de comorbilidad el $68 \%$ de los pacientes. El 38\% de los pacientes tomaban AINE bien de forma continua o intermitente y el $13 \%$ estaban anticoagulados, con un INR medio al ingreso de 1,3 $(1,0-5,7)$. El sangrado se acompañó de episodio sincopal en el 9\% de los casos. Tenían antecedentes de HDA previa el $11 \%$ de los pacientes y el $31 \%$ habían presentado al menos un episodio previo de HDB. Fue precisa la transfusión de hemoderivados en el $36 \%$ de los pacientes. Estos y otros hallazgos se hallan recogidos en la tabla I.

\begin{tabular}{|c|c|c|c|}
\hline jun & $\begin{array}{c}\text { Retrospectivo } \\
\text { unio 2005-junio } 2006 \\
n=137\end{array}$ & $\begin{array}{c}\text { Prospectivo } \\
\text { julio 2006-julio } 2007 \\
n=96\end{array}$ & $p$ \\
\hline Edad (años) & $73,5 \pm 13$ & $71,3 \pm 20$ & NS \\
\hline Mujeres & $71(52 \%)$ & $50(52 \%)$ & NS \\
\hline Comorbilidad & $94(69 \%)$ & $75(78 \%)$ & NS \\
\hline tábito tabáquico (cig./día) & $12(9 \%)$ & $15(16 \%)$ & NS \\
\hline Hábito etilico > 20 g/día & $12(8,8 \%)$ & $3(3 \%)$ & NS \\
\hline ngesta AINE & $52(38 \%)$ & $30(31 \%)$ & NS \\
\hline ratamiento anticoagulante & $18(13 \%)$ & $19(19 \%)$ & NS \\
\hline Dolor abdominal & $43(31 \%)$ & $43(45 \%)$ & NS \\
\hline Alteración ritmo intestinal & $40(29 \%)$ & $39(41 \%)$ & $<0,05$ \\
\hline Síndrome constitucional & $11(8 \%)$ & $18(19 \%)$ & NS \\
\hline Síncope & $13(9 \%)$ & $8(8 \%)$ & NS \\
\hline Transfusión de hemoderivados & $50(36 \%)$ & $40(42 \%)$ & NS \\
\hline $\begin{array}{l}\text { Hemoglobina al ingreso } \\
(\mathrm{g} / \mathrm{dl}) \\
\text { Hemoglobina al alta }\end{array}$ & $11,5 \pm 3$ & $11,2 \pm 3$ & NS \\
\hline$(g / d)$ & $11,4 \pm 3$ & $12 \pm 5$ & NS \\
\hline NR al ingreso & $1,3 \pm 0,8$ & $1,5 \pm 1$ & NS \\
\hline HDA previa & $15(11 \%)$ & $17(18 \%)$ & NS \\
\hline ADB previa & $43(31 \%)$ & $32(33 \%)$ & NS \\
\hline
\end{tabular}

AINE: antiinflamatorios no esteroideos; HDA: hemorragia digestiva alta; HDB: hemorragia digestiva baja; NS: no significativo.

La inspección anal fue normal en el 83\% de las exploraciones. Se objetivó fisura anal en 3 pacientes y patología hemorroidal en 19. En ninguno de estos casos se pudo atribuir el sangrado a dichos hallazgos. En el tacto rectal en el 25\% de los casos no se objetivó sangrado, en el $47 \%$ se halló sangre roja, en el $18 \%$ hematoquecia y en el $9 \%$ melenas.

Se realizó colonoscopia a 106 pacientes; se hallaron divertículos en el $27 \%$ siendo la causa más frecuente de HDB seguido por las neoplasias en el $12 \%$ y en tercer lugar las angiodisplasias en el 5\% de los pacientes. En el $9 \%$ de los casos no se encontraron hallazgos en la colonoscopia. En el 4\% de los casos el sangrado se atribuyó a patología anorrectal diagnosticada por anuscopia. La mayoría de las lesiones se situaron en colon izquierdo (28\%) y recto $(15 \%)$. Estos y otros hallazgos se hallan recogidos en la tabla II. 
Tabla II. Hallazgos en el tacto rectal. Colonoscopia: hallazgos y localización

\begin{tabular}{lccc}
\hline Variable & $\begin{array}{c}\text { Retrospectivo } \\
\text { junio } 2005 \text {-junio } 2006 \\
n=137\end{array}$ & $\begin{array}{c}\text { Prospectivo } \\
\text { julio 2006-julio } 2007 \\
n=96\end{array}$ & $p$ \\
\hline Hallazgos tacto rectal & & & \\
Normal & $35(25 \%)$ & $12(12 \%)$ & NS \\
Sangre roja & $65(47 \%)$ & $64(67 \%)$ & NS \\
Hematoquecia & $25(18 \%)$ & $12(12 \%)$ & NS \\
Melena & $12(9 \%)$ & $8(8 \%)$ & NS \\
Hallazgos colonoscopia & & & \\
Divertículos & $38(35 \%)$ & $30(39 \%)$ & NS \\
Neoplasias & $17(15 \%)$ & $12(16 \%)$ & NS \\
Sin hallazgos & $13(12 \%)$ & $10(13 \%)$ & NS \\
Angiodisplasias & $7(7 \%)$ & $3(4 \%)$ & NS \\
Colitis isquémica & $6(6 \%)$ & $5(6 \%)$ & NS \\
& & & \\
Localización lesión & $60(63 \%)$ & $41(64 \%)$ & NS \\
Colon izquierdo-sigma & $22(22 \%)$ & $11(17 \%)$ & NS \\
Recto & $7(7 \%)$ & $7(11 \%)$ & NS \\
Colon derecho & $7(7 \%)$ & $5(8 \%)$ & NS \\
Colon transverso & & & \\
\hline
\end{tabular}

NS: no significativo.

Se llevó a cabo terapia endoscópica en 16 casos; en 2 mediante inyección de sustancias esclerosantes, en 8 se utilizó argón-plasma, en 4 electrotermocoagulación y en 2 casos se colocaron hemoclips.

El tiempo transcurrido desde el ingreso hasta la realización de la colonoscopia fue de 4,1 (0-23) días, siendo el 0 el valor atribuido a aquellas que se realizaron el mismo día del ingreso de forma urgente. De los 137 pacientes, el $62 \%$ presentaron una evolución favorable, el $2 \%$ tuvieron un sangrado persistente y el $18 \%$ recurrente. Estos hallazgos se hallan recogidos en la tabla III.

Tabla III. Evaluación, tiempo de espera para la realización de la colonoscopia y estancia media hospitalaria

\begin{tabular}{lccc}
\hline Variable & $\begin{array}{c}\text { Retrospectivo } \\
\text { junio } 2005 \text {-junio } 2006 \\
n=137\end{array}$ & $\begin{array}{c}\text { Prospectivo } \\
\text { julio 2006-julio 2007 } \\
n=96\end{array}$ & $p$ \\
\hline Evolución & & & \\
Favorable & $85(62 \%)$ & $65(68 \%)$ & $\mathrm{NS}$ \\
Persistencia & $3(2 \%)$ & $4(4 \%)$ & $\mathrm{NS}$ \\
Recurrencia & $25(18 \%)$ & $15(16 \%)$ & $\mathrm{NS}$ \\
Cirugía & $15 / 137(10 \%)$ & $4 / 96(4 \%)$ & $<0,05$ \\
Embolización & $1(0,7 \%)$ & $3(3 \%)$ & $\mathrm{NS}$ \\
Éxitus & $5(4 \%)$ & $2(2 \%)$ & $\mathrm{NS}$
\end{tabular}

Tiempo de espera para colonoscopia (días)

$$
4,15 \pm 3,6 \quad 2,6 \pm 2,4<0,05
$$$$
\text { Estancia media (días) } \quad 10,21 \pm 6,1 \quad 7,7 \pm 5,2<0,05
$$

NS: no significativo.
Catorce pacientes fueron derivados al Servicio de cirugía por lesiones neoplásicas; ninguno de los 137 pacientes requirió cirugía urgente por imposibilidad de control del sangrado mediante otras técnicas. Un paciente requirió embolización vascular guiada por técnicas radiológicas y en 5 pacientes se produjo el éxitus.

\section{Estudio prospectivo: 2006-2007}

De los 96 pacientes incluidos 46 eran hombres y 50 mujeres. La edad media fue de 71,3 (20-97) años. Presentaron datos de comorbilidad el $78 \%$ de los pacientes. Refirieron ingesta de AINE el 31 y el 20\% estaban anticoagulados. Presentaban antecedentes de HDA el 18 y el $33 \%$ habían presentado al menos un episodio previo de HDB. Precisaron transfusión de hemoderivados el $42 \%$ de los pacientes.

La inspección anal fue normal en el 83\% de las exploraciones, se objetivó fisura anal en 1 paciente, fístula anal en 5 y patología hemorroidal en 10; en ninguno de los casos se atribuyó la HDB a estos hallazgos. En el tacto rectal en el $12 \%$ de los pacientes no se objetivó sangrado, en el $67 \%$ se halló sangre roja, en el $13 \%$ hematoquecia y en el $8 \%$ melenas.

Se realizó colonoscopia al 80\%; se hallaron divertículos en el 31,3\%, siendo este el hallazgo más frecuente, seguido de angiodisplasias (12\%) y en tercer lugar de colitis isquémica $(5 \%)$. El $6 \%$ se atribuyó a patología anorrectal y en $10(10,4 \%)$ de los casos no hubo hallazgos en la colonoscopia. La mayoría de las lesiones se situaron en sigma (29\%). Estos y otros hallazgos se encuentran recogidos en la tabla II.

Se realizó terapia endoscópica en 6 casos: en 2 pacientes se inyectaron sustancias esclerosantes, en 3 se utilizó argón-plasma, en 4 electrotermocoagulación y en 2 hemoclips. El tiempo transcurrido desde el ingreso hasta la realización de la colonoscopia fue de 2,6 (0-10) días.

Presentaron evolución favorable el $68 \%$ de los pacientes, el $4 \%$ persistente y el $16 \%$ recurrente. Fueron derivados al Servicio de Cirugía General 4 casos por patología neoplásica y 3 requirieron embolización vascular mediante técnicas radiológicas para conseguir el control de la hemorragia. En 2 pacientes se produjo el éxitus. Estos y otros hallazgos se hallan recogidos en la tabla III.

Ninguna de las variables estudiadas demostró asociación lineal con el tiempo de estancia media.

\section{DISCUSIÓN}

Existen muchas controversias acerca del manejo de la HDB en pacientes hospitalizados, no existiendo un consenso acerca de los criterios de ingreso, ni tampoco en los posibles factores que puedan influir en su presentación y evolución. A pesar de que la mayoría de las HDB tienen un curso favorable $(2-4,7)$, los médicos continuamos in- 
gresando casi de forma sistemática a todo paciente que acude a Urgencias por este motivo, incrementando probablemente el gasto sanitario, así como la posibilidad de sufrir complicaciones derivadas de la hospitalización, sobre todo en pacientes ancianos. Este hecho fue lo que motivó la realización del presente estudio.

La incidencia de HDB es mayor en pacientes varones, ancianos y con comorbilidad asociada $(2,4)$. En nuestro trabajo la mayoría de los pacientes eran mayores de 70 años y presentaban comorbilidad aunque, a diferencia de lo descrito en la literatura en nuestra serie la prevalencia fue similar en ambos sexos. La etiología más frecuente de HDB en ambos grupos fueron los divertículos cólicos en concordancia con estudios previos $(4-6,12,19)$ pero encontramos que las neoplasias y no las angiodisplasias fueron la segunda causa más frecuente al contrario que lo que describen otros autores $(2,4,8)$.

Una cuestión importante es la precocidad de la realización de la colonoscopia. ¿La colonoscopia realizada de forma más temprana aportaría un mayor número de diagnósticos? En este estudio se modificó la pauta de actuación ante una HDB tras el primer periodo estudiado (2005-2006), tratando de reducir al máximo posible el tiempo de espera de la colonoscopia. Así, se pasó de 4,1 días de media en el estudio retrospectivo a 2,6 días de media en el prospectivo; sin embargo, el porcentaje de colonoscopias no diagnósticas fue prácticamente igual en ambos. Por tanto, no se logró mejorar el rendimiento diagnóstico del procedimiento. Diversos estudios han demostrado una reducción de la estancia media hospitalaria en relación con una colonoscopia precoz $(13,19,20)$ pero, sólo se ha logrado mejorar el rendimiento diagnóstico endoscópico cuando esta se realiza en las 6-8 primeras horas (19).

Ambos grupos fueron homogéneos para las variables estudiadas excepto en las alteraciones en el tránsito intestinal en los 6 meses previos al ingreso y en el número de casos en los que fue necesaria la cirugía; esto se podría justificar porque la cirugía realizada fue oncológica; en realidad ningún paciente precisó cirugía urgente por sangrado masivo, ya que estos casos fueron solucionados mediante tratamiento angiográfico con embolización supraselectiva. Debido a que cuando se realizó el estudio prospectivo disponíamos de una mayor accesibilidad a la colonoscopia, muchos de los pacientes con sospecha de patología neoplásica vistos en Urgencias fueron dados de alta con la certeza de que se les realizaría una endoscopia precoz, de forma que no hubo una disminución real de la patología tumoral, sino un mayor número de pacientes diagnosticados de forma ambulatoria. No obstante, no encontramos diferencias estadísticamente significativas en el número de tumores diagnosticados entre ambos periodos estudiados. Será necesario un análisis interno de los datos con mayor precisión para explicar este fenómeno.

Finalmente reseñaremos, que el cambio en el manejo de la HDB, a expensas sobre todo de un mayor apoyo de la Unidad de Endoscopias permitió reducir el tiempo de espera para la realización de la colonoscopia en un $36 \%$ y que la estancia media se redujo en un $24 \%$ entre ambos periodos. Podemos por tanto concluir que la colonoscopia precoz disminuye el tiempo de estancia media hospitalaria (hecho ya constatado en estudios previos) y por tanto una reducción en el gasto sanitario y una mejor calidad asistencial a los pacientes ingresados por HDB. Sin embargo la realización más temprana de la colonoscopia no permitió obtener un mayor número de diagnósticos ni una disminución de los casos de recurrencia de HDB.

\section{BIBLIOGRAFÍA}

1. Peura DA, Lanza FL, Gostout CI, Foutch PG. The American College of Gastroenterology Bleeding Registry: Preliminary findings. Am J Gastroenterology 1997; 92: 924.

2. Farrek JJ, Friedman LS. The management of lower gastrointestinal bleeding: rewire article. Aliment Pharmacol Ther 2005; 21: 1281-98.

3. Saperas E. Lower gastrointestinal bleeding: the great unknown. Gastroenterol Hepatol 2007; 30: 93-100.

4. Edelman DA, Sugawa C. Lower gastrointestinal bleeding: a review. Surg Endosc 2007; 21: 514-20.

5. Rockey DC. Lower gastrointestinal bleeding. Gastroenterology 2006; 130: 165-71.

6. Ríos A, Montoya JM. Severe acute lower gastrointestinal bleeding: risk factors for morbidity and mortality. Langenbecks Arch Surg 2007; 392: 165-71.

7. Strate LL, Orav EJ. Early predictors of severity in acute lower intestinal tract bleeding. Arch Intern Med 2003; 163: 838-43.

8. Ritchter JM, Christensen MR. Effectiveness of current technology in the diagnosis and management of lower gastrointestinal hemorrhage. Gastointest Endosc 1995; 41: 93.

9. Gibbs DH, Opelka FG. Postpolypectomy colonic hemorrhage. Dis Colon Rectum 1996; 39: 806-10.

10. Sieg A, Eisenbach H, Eisenbach T. Prospective evaluation of complications in outpatient GI endoscopy a survey among German gastroenterologists. Gastrointest Endosc 2001; 53: 620-7.

11. Kim HS, Kim TI. Risk factors for immediate postpolypectomy bleeding of the colon: a multicenter study. Am J Gastroenterol 2006; 101: 1333-41.

12. Lin CC, Wang HP. The etiology and clinical characteristics of acute lower gastrointestinal bleeding in patients hospitalized for comorbid illnesses. Hepatogastroenterology 2006; 53: 395-8.

13. Strate LL, Syngal S. Timing of colonoscopy: impact on length of hospital stay in patients with acute lower intestinal bleeding. Am J Gastroenterol 2003; 98: 317-22.

14. Green BT, Rockey DC. Urgent colonoscopy for evaluation and management of acute lower gastrointestinal haemorrhage: a randomized controlled trial. Am J Gastroenterol 2005; 100: 2395-402.

15. Strate LL, Syngal S. Predictors of utilization of early colonoscopy vs radiography for severe lower intestinal bleeding. Gastrointest Endosc 2005; 61: 46-52.

16. American Society for Gastrointestinal Endoscopy: the role of endoscopy in the patient with lower gastrointestinal bleeding. Gastrointest Endosc 1998; 48: 685 .

17. Padia SA, Bybel B. Radiologic diagnosis and management of acute lower gastrointestinal bleeding. Cleve Clin J Med 2007; 74: 417-20.

18. Laine L, Smith R. Systematic review: the lower gastrointestinal adverse effects of non-steroidal anti-inflammatory drugs. Aliment Pharmacol Ther 2006; 24: 751-67.

19. Sánchez A, Muñoz C, Bujanda L, Iriondo C, Gil-Molet A, Cosme A, et al. The value of colonoscopy to assess rectal bleeding in patients referred from primare care unit. Rev Esp Enferm Dig 2005; 97: 870-6.

20. García Sánchez MV, González Galilea A, López Vallejos, Gálvez Calderón A, Naranjo Rodríguez J, De dios Vega J, et al. Role of early colonoscopy in severe acute lower gastrointestinal bleeding. Gastroenterol Hepatol 2001; 24: 327-32. 